A Finite-Difference Numerical Method for Onsager's Pancake Approximation for Fluid Flow in a Gas Centrifuge

M. de Stadler, K. Chand

November 15, 2007 
This document was prepared as an account of work sponsored by an agency of the United States government. Neither the United States government nor Lawrence Livermore National Security, LLC, nor any of their employees makes any warranty, expressed or implied, or assumes any legal liability or responsibility for the accuracy, completeness, or usefulness of any information, apparatus, product, or process disclosed, or represents that its use would not infringe privately owned rights. Reference herein to any specific commercial product, process, or service by trade name, trademark, manufacturer, or otherwise does not necessarily constitute or imply its endorsement, recommendation, or favoring by the United States government or Lawrence Livermore National Security, LLC. The views and opinions of authors expressed herein do not necessarily state or reflect those of the United States government or Lawrence Livermore National Security, LLC, and shall not be used for advertising or product endorsement purposes.

This work performed under the auspices of the U.S. Department of Energy by Lawrence Livermore National Laboratory under Contract DE-AC52-07NA27344. 


\title{
A Finite-Difference Numerical Method for Onsager's Pancake Approximation for Fluid Flow in a Gas Centrifuge
}

\author{
Matthew de Stadler \\ Lawrence Livermore National Laboratory \\ Kyle Chand* \\ Lawrence Livermore National Laboratory
}

November 12, 2007

\section{Contents}

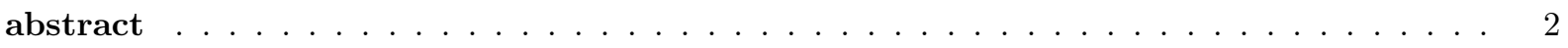

1 Introduction 2

2 Equations of Motion in Axisymmetric Coordinates 2

2.1 Wheel Flow Equilibrium Solution $\ldots \ldots \ldots \ldots \ldots$

3 Linearizing the Equations of Motion $\quad 3$

3.1 Linearizing Transformations . . . . . . . . . . . . . . . . . . . . . . . 3

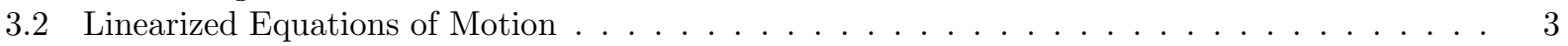

3.3 Deriving Onsager's Master Equation . . . . . . . . . . . . . . . . . . . . . . 4

3.4 Non-Dimensional Form of Onsager's Master Equation _ . . . . . . . . . . . . . . . . . . 6

4 Solving Onsager's Master Equation $\quad 7$

4.1 Coupled System of Partial Differential Equations _ . . . . . . . . . . . . . . . . . . 7

4.2 Boundary Conditions: Total Reflux Case . . . . . . . . . . . . . . . . . . . . 8

4.3 Problem Parameters . . . . . . . . . . . . . . . . . . . . . . . 8

4.4 Choosing A Suitable Grid $\ldots \ldots \ldots \ldots \ldots \ldots$

5 Results 9

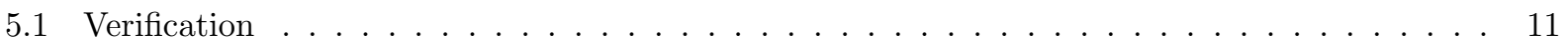

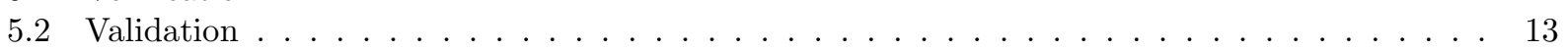

5.3 Known Errors . . . . . . . . . . . . . . . . . . . . . . . . . . . . . . . 13

6 Conclusion $\quad 13$

7 Future Work $\quad 16$

$\begin{array}{ll}\text { A Pseudocode for Simulation } & 16\end{array}$

${ }^{*}$ For correspondence on this paper contact Kyle Chand at chand1@llnl.gov 


\begin{abstract}
Onsager's pancake approximation is used to construct a simulation to model fluid flow in a gas centrifuge. The governing 6th order partial differential equation is broken down into an equivalent coupled system of three equations and then solved numerically. In addition to a discussion on the baseline solution, known problems and future work possibilities are presented.
\end{abstract}

\title{
1 Introduction
}

Gas centrifuges exhibit very complex flows. Within the centrifuge there is a rarefied region, a transition region, and a region with an extreme density gradient. The flow moves at hypersonic speeds and shock waves are present. However, the flow is subsonic in the axisymmetric plane.

The analysis may be simplified by treating the flow as a perturbation of wheel flow. Wheel flow implies that the fluid is moving as a solid body. With the very large pressure gradient, the majority of the fluid is located very close to the rotor wall and moves at an azimuthal velocity proportional to its distance from the rotor wall; there is no slipping in the azimuthal plane. The fluid can be modeled as incompressible and subsonic in the axisymmetric plane. By treating the centrifuge as long, end effects can be appropriately modeled without performing a detailed boundary layer analysis.

\section{Equations of Motion in Axisymmetric Coordinates}

The equations of motion for a steady-state axisymmetric fluid in cylindrical coordinates are as follows. It is assumed that temperature differences in the fluid are minimal which results in a constant viscosity, constant specific heat, and constant thermal conductivity of the gas. These equations are the starting point for the analysis of fluid flow in a gas centrifuge.

Mass continuity:

$$
\frac{1}{r} \frac{\partial}{\partial r}\left(\rho r V_{r}\right)+\frac{\partial}{\partial z}\left(\rho V_{z}\right)=0
$$

Axial momentum:

$$
\rho\left(V_{r} \frac{\partial V_{z}}{\partial r}+V_{z} \frac{\partial V_{z}}{\partial z}\right)=-\frac{\partial p}{\partial z}+\mu\left\{\frac{1}{r} \frac{\partial}{\partial r}\left(r \frac{\partial V_{z}}{\partial r}\right)+\frac{\partial^{2} V_{z}}{\partial z^{2}}\right\}+\rho g
$$

Radial momentum:

$$
\rho\left(V_{r} \frac{\partial V_{r}}{\partial r}-\frac{V_{\theta}^{2}}{r}+V_{z} \frac{\partial V_{r}}{\partial z}\right)=-\frac{\partial p}{\partial r}+\mu\left\{\frac{\partial}{\partial r}\left[\frac{1}{r} \frac{\partial}{\partial r}\left(r V_{r}\right)\right]+\frac{\partial^{2} V_{r}}{\partial z^{2}}\right\}
$$

Azimuthal momentum:

$$
\rho\left(V_{r} \frac{\partial V_{\theta}}{\partial r}+\frac{V_{r} V_{\theta}}{r}+V_{z} \frac{\partial V_{\theta}}{\partial z}\right)=\mu\left\{\frac{\partial}{\partial r}\left[\frac{1}{r} \frac{\partial}{\partial r}\left(r V_{\theta}\right)\right]+\frac{\partial^{2} V_{\theta}}{\partial z^{2}}\right\}
$$

Energy equation:

$$
\rho C_{v}\left(V_{r} \frac{\partial T}{\partial r}+V_{z} \frac{\partial T}{\partial z}\right)+T\left(\frac{\partial p}{\partial T}\right)_{\rho}\left[\frac{1}{r} \frac{\partial}{\partial r}\left(r V_{r}\right)+\frac{\partial V_{z}}{\partial z}\right]=\kappa\left[\frac{1}{r} \frac{\partial}{\partial r}\left(r \frac{\partial T}{\partial r}\right)+\frac{\partial^{2} T}{\partial z^{2}}\right]
$$

where $r$ is the radius, $\rho$ the density, $p$ the pressure, $T$ the temperature, $\mu$ the viscosity, $g$ gravity, $C_{v}$ the specific heat at constant volume of the gas, and $\kappa$ the thermal conductivity of the gas. $V_{r}, V_{z}$, and $V_{\theta}$ are the velocity components in the radial, axial, and azimuthal directions, respectively.

is this section indented... how do I fix this? 
Equation of state for an ideal gas:

$$
p=\rho \bar{R} T
$$

where $\mathrm{R}$ is the general gas constant, $\mathrm{M}$ the molecular weight and $\bar{R}$ is defined as follows

$$
\bar{R}=\frac{R}{M}
$$

\subsection{Wheel Flow Equilibrium Solution}

It is helpful to construct a solution for the case when the fluid in the gas centrifuge is in equilibrium. This occurs in the ideal case where flow perturbations due to thermal or mechanical effects do not exist.

$$
\begin{aligned}
V_{r} & =0 \\
V_{\theta} & =\Omega r \\
V_{z} & =0 \\
T & =T_{0} \\
\left(\frac{\partial p}{\partial z}\right)_{0}=\rho_{0} g & \approx 0 \\
\left(\frac{\partial p}{\partial r}\right)_{0}=\rho_{0} \Omega^{2} r & =\frac{p_{0}}{\bar{R} T_{0}} \Omega^{2} r
\end{aligned}
$$

where $\Omega$ is the rotation rate of the cylinder wall. Integration of Equation 8 results in

$$
\begin{aligned}
& p_{0}(r)=p(0) \exp \left[\left(M \Omega^{2} / 2 R T_{0}\right) r^{2}\right] \\
& \rho_{0}(r)=\rho(0) \exp \left[\left(M \Omega^{2} / 2 R T_{0}\right) r^{2}\right]
\end{aligned}
$$

\section{Linearizing the Equations of Motion}

\subsection{Linearizing Transformations}

Each of the problem relevant primitive variables are written as a combination of an equilibrium value and a perturbation value.

$$
\begin{array}{cr}
V_{z}=0+u & \tilde{\rho}=\rho_{0}+\rho \\
V_{r}=0+v & \tilde{p}=p_{0}+p \\
V_{\theta}=\Omega r+w & \tilde{T}=T_{0}+T
\end{array}
$$

where $u, v$, and $w$ represent the perturbation velocities in the axial, radial, and azimuthal direction. Perturbations of the gas due to internal circulation are given by $p, \rho$, and $T$.

\subsection{Linearized Equations of Motion}

After applying the linearizing transformations and neglecting the products of perturbation values, also neglecting the effect of gravity due to its relatively small impact compared to the centrifugal force term, the 
following linearized equations of motion are obtained.

Mass continuity:

$$
\frac{1}{r} \frac{\partial}{\partial r}\left(\rho_{0} v r\right)+\frac{\partial}{\partial z}\left(\rho_{0} u\right)=0
$$

Axial momentum:

$$
0=-\frac{\partial p}{\partial z}+\mu\left\{\frac{1}{r} \frac{\partial}{\partial r}\left(r \frac{\partial u}{\partial r}\right)+\frac{\partial^{2} u}{\partial z^{2}}\right\}
$$

Radial momentum:

$$
-\rho r \Omega^{2}-2 \rho_{0} \Omega w=-\frac{\partial p}{\partial r}+\mu\left\{\frac{\partial}{\partial r}\left[\frac{1}{r} \frac{\partial}{\partial r}(r v)\right]+\frac{\partial^{2} v}{\partial z^{2}}\right\}
$$

Azimuthal momentum:

$$
2 \rho_{0} \Omega v=\mu\left\{\frac{\partial}{\partial r}\left[\frac{1}{r} \frac{\partial}{\partial r}(r w)\right]+\frac{\partial^{2} w}{\partial z^{2}}\right\}
$$

Energy equation:

$$
-\rho_{0} \Omega^{2} r v=\kappa\left[\frac{1}{r} \frac{\partial}{\partial r}\left(r \frac{\partial T}{\partial r}\right)+\frac{\partial^{2} T}{\partial z^{2}}\right]
$$

Equation of state for an ideal gas:

$$
\rho=\frac{p}{T_{0} \bar{R}}-\rho_{0} \frac{T}{T_{0}}
$$

\subsection{Deriving Onsager's Master Equation}

Beginning with the linearized equations of motion, a master equation is derived. In addition to the following description, one may also consult Appendix A of Olander's Theory of Uranium Enrichment by the Gas Centrifuge[1]. The general strategy for this derivation is as follows:

From the basic linearized equations there are six variables: $\rho, \mathrm{p}, \mathrm{T}, \mathrm{u}, \mathrm{v}, \mathrm{w}$. The goal is to reduce terms until only one is left.

1. Drop all terms in Equations 11-16 involving axial diffusion of heat and momentum and the term containing radial diffusion of radial momentum.

$$
\begin{aligned}
\frac{1}{r} \frac{\partial}{\partial r}\left(\rho_{0} v r\right)+\frac{\partial}{\partial z}\left(\rho_{0} u\right) & =0 \\
0 & =-\frac{\partial p}{\partial z}+\mu\left\{\frac{1}{r} \frac{\partial}{\partial r}\left(r \frac{\partial u}{\partial r}\right)\right\} \\
-\rho r \Omega^{2}-2 \rho_{0} \Omega w & =-\frac{\partial p}{\partial r} \\
2 \rho_{0} \Omega v & =\mu\left\{\frac{\partial}{\partial r}\left[\frac{1}{r} \frac{\partial}{\partial r}(r w)\right]\right) \\
-\rho_{0} \Omega^{2} r v & =\kappa\left[\frac{1}{r} \frac{\partial}{\partial r}\left(r \frac{\partial T}{\partial r}\right)\right] \\
\rho & =\frac{p}{T_{0} \bar{R}}-\rho_{0} \frac{T}{T_{0}}
\end{aligned}
$$


2. Substitute Equation 16 into Equation 13 to eliminate $\rho$ as a variable. Also divide by $\rho_{0} r$.

$$
\Omega\left(\frac{-T}{T_{0}} \Omega+\frac{2 w}{r}\right)=\frac{1}{r \rho_{0}} \frac{\partial p}{\partial r}-\frac{p \Omega^{2}}{\rho_{0} R T_{0}}
$$

3. Rewrite the right hand side of Equation 21 using $\frac{\partial p}{\partial r}=\rho_{0} \frac{\partial}{\partial r}\left(\frac{p}{\rho_{0}}\right)+p\left(\frac{\Omega^{2} r}{R T_{0}}\right)$.

$$
\Omega\left(\frac{-T}{T_{0}} \Omega+\frac{2 w}{r}\right)=\frac{1}{r} \frac{\partial}{\partial r}\left(\frac{p}{\rho_{0}}\right)
$$

4. Take $\frac{\partial}{\partial z}$ of the Equation 22 .

$$
\Omega \frac{\partial}{\partial z}\left(\frac{-T}{T_{0}} \Omega+\frac{2 w}{r}\right)=\frac{1}{r} \frac{\partial}{\partial r} \frac{\partial}{\partial z}\left(\frac{p}{\rho_{0}}\right)
$$

5. Combine Equation 22 with Equation 17 to remove $p$ as a variable. Note that $\rho_{0}$ has no axial dependence.

$$
\Omega \frac{\partial}{\partial z}\left(\frac{-T}{T_{0}} \Omega+\frac{2 w}{r}\right)=\frac{1}{r} \frac{\partial}{\partial r} \frac{1}{\rho_{0}}\left[\mu \frac{1}{r} \frac{\partial}{\partial r}\left(r \frac{\partial u}{\partial r}\right)\right]
$$

6. Introduce a stream function, $\psi$, and master potential function, $\chi$ as follows

$$
\begin{array}{r}
r \rho_{0} v=-\frac{\partial \psi}{\partial z}=-\frac{1}{r} \frac{\partial}{\partial r} \frac{\partial \chi}{\partial z} \\
\rho_{0} u=-\frac{1}{r} \frac{\partial \psi}{\partial r}=-\frac{1}{r} \frac{\partial}{\partial r}\left(\frac{1}{r} \frac{\partial \chi}{\partial r}\right)
\end{array}
$$

7. Take $\frac{1}{r} \frac{\partial}{\partial r}$ of Equation 24 and substitute in Equation 26 to remove $u$.

$$
\frac{\Omega^{2}}{r} \frac{\partial^{2}}{\partial r \partial z}\left(\frac{-T}{T_{0}}+\frac{2 w}{\Omega r}\right)=\frac{\partial^{2}}{(r \partial r)^{2}}\left[\frac{\mu \rho_{0}}{r} \frac{\partial}{\partial r}\left\{r \frac{\partial}{\partial r}\left(\frac{1}{\rho_{0}} \frac{\partial^{2} \chi}{(r \partial r)^{2}}\right)\right\}\right]
$$

8. Manipulate Equation 14 with the identity $\frac{1}{r^{2}} \frac{\partial}{\partial r}\left[r^{3} \frac{\partial}{\partial r}\left(\frac{w}{r}\right)\right]=\frac{\partial}{\partial r}\left[\frac{1}{r} \frac{\partial}{\partial r}(w r)\right]$

$$
2 \rho_{0} \Omega v=\frac{\mu}{r^{2}} \frac{\partial}{\partial r}\left[r^{3} \frac{\partial}{\partial r}\left(\frac{w}{r}\right)\right]
$$

9. Multiply Equation 25 by $\frac{2 \Omega}{r}$ and combine with Equation 28, divide by $r$, move $\mu, r^{3}$ to the left. This removes $v$.

$$
\frac{-2 \Omega}{\mu r^{4}} \frac{\partial \chi}{\partial z}=\frac{1}{r} \frac{\partial}{\partial r}\left(\frac{w}{r}\right)
$$

10. Multiply Equation 25 by $\Omega^{2}$ and combine with Equation 15 .

$$
r \frac{\partial}{\partial r}\left(\frac{T}{T_{0}}\right)=\frac{\Omega^{2}}{\kappa T_{0}} \frac{\partial \chi}{\partial z}
$$

11. Substitute Equation 30 into the first term of the left hand side of Equation 27. Substitute Equation 29 into the second term of the left hand side of Equation 27. This removes $T$ and $w$ making $\chi$ the sole remaining variable.

First term on the left hand side of Equation 27.

$$
-\frac{\Omega^{2}}{r} \frac{\partial^{2}}{\partial r \partial z}\left(\frac{T}{T_{0}}\right)=-\frac{\Omega^{2}}{r} \frac{\partial}{\partial z}\left[r \frac{\partial}{\partial r}\left(\frac{T}{T_{0}}\right)\right]
$$


Second term on the left hand side of Equation 27.

$$
\frac{2 \Omega}{r} \frac{\partial^{2}}{\partial r \partial z}\left(\frac{w}{r}\right)=2 \Omega \frac{\partial}{\partial z}\left[\frac{1}{r} \frac{\partial}{\partial r}\left(\frac{w}{r}\right)\right]
$$

Left hand side of Equation 27 after substitution.

$$
-\frac{\Omega^{2}}{r^{2}} \frac{\partial}{\partial z}\left(\frac{\Omega^{2}}{T_{0} \kappa} \frac{\partial \chi}{\partial z}\right)+2 \Omega \frac{\partial}{\partial z}\left(\frac{-2 \Omega}{\mu r^{4}} \frac{\partial \chi}{\partial z}\right)
$$

12. Clean up and collect terms to write the solution.

Onsager's Master Equation in dimensional form:

$$
\frac{\partial^{2}}{(r \partial r)^{2}}\left\{\frac{1}{\rho_{0}} \frac{1}{r} \frac{\partial}{\partial r}\left[r \frac{\partial}{\partial r}\left(\frac{1}{\rho_{0}} \frac{\partial^{2} \chi}{(r \partial r)^{2}}\right)\right]\right\}+\frac{1}{\mu}\left(\frac{4 \Omega^{2}}{\mu r^{4}}+\frac{\Omega^{4}}{\kappa T_{0} r^{2}}\right) \frac{\partial^{2} \chi}{\partial z^{2}}=0
$$

note that:

$$
\frac{\partial^{2}}{(r \partial r)^{2}}=\frac{1}{r} \frac{\partial}{\partial r}\left(\frac{1}{r} \frac{\partial}{\partial r}\right)
$$

\subsection{Non-Dimensional Form of Onsager's Master Equation}

By introducing the non-dimensional variables $\xi$ and $\eta$, Onsager's Master Equation can be non-dimensionalized.

$$
\begin{array}{r}
\xi=A^{2}\left(1-\frac{r^{2}}{a^{2}}\right) \\
\eta=\frac{z}{Z}
\end{array}
$$

Substituting Equation 35 and Equation 36 into Equation 34 and taking advantage of the following useful equations to collect terms, one is able to obtain the non-dimensionalized form. The assumption $1-\frac{\epsilon}{A^{2}} \approx 1$ is required to obtain the simplified form.

$$
\begin{aligned}
\frac{1}{\rho_{0}} & =\frac{e^{\xi}}{\rho_{w}} \\
\operatorname{Pr} & =\frac{\mu c_{p}}{\kappa M} \\
A^{2} & =\frac{M \Omega^{2} a^{2}}{2 R T_{0}} \\
\frac{C_{p}}{R} & =\frac{\gamma}{\gamma-1} \\
\epsilon & =\frac{\mu}{\rho_{w} \Omega a^{2}}
\end{aligned}
$$

where Pr is the Prandtl number and $\gamma$ is the ratio of specific heats.

Non-Dimensionalized Version of Onsager's Master Equation:

$$
\frac{\partial^{2}}{\partial \xi^{2}} e^{\xi} \frac{\partial^{2}}{\partial \xi^{2}} e^{\xi} \frac{\partial^{2} \chi}{\partial \xi^{2}}+\frac{1+A^{2} \operatorname{Pr} \frac{\gamma-1}{2 \gamma}}{16 A^{12} \epsilon^{2}\left(\frac{Z}{a}\right)^{2}} \frac{\partial^{2} \chi}{\partial \eta^{2}}=0
$$

Note that in this format, $\chi$ has units of $\frac{\text { MassLength }}{2}$. To completely non-dimensionalize, divide $\chi$ by $\rho_{w} \Omega a^{5}$. 


\section{Solving Onsager's Master Equation}

\subsection{Coupled System of Partial Differential Equations}

Rather than attempt to solve the full sixth order partial differential equation as written above, an equivalent coupled system of three second order partial differential equations is written. The system is given as follows

$$
\begin{aligned}
\frac{\partial^{2} \varrho}{\partial \xi^{2}}+\frac{1+A^{2} \operatorname{Pr} \frac{\gamma-1}{2 \gamma}}{16 A^{12} \epsilon^{2}\left(\frac{Z}{a}\right)^{2}} \frac{\partial^{2} \chi}{\partial \eta^{2}} & =0 \\
\frac{\partial^{2} \varphi}{\partial \xi^{2}}-e^{-\xi} \varrho & =0 \\
\frac{\partial^{2} \chi}{\partial \xi^{2}}-e^{-\xi} \varphi & =0
\end{aligned}
$$

There are several advantages to formulating the equation this way.

1. A more compact stencil is allowed

2. Allows more general cases to be considered than an eigenfunction expansion

3. Boundary Conditions are easier to implement in this formulation

4. More direct formulation when used with Overture

5. Easier to implement in Overture

To solve a sixth order PDE with second order accuracy would require a stencil with a width of seven grid points. The system of second order equations requires a stencil with a width of three grid points. For fourth order accuracy with this setup, a stencil width of five grid points is required. The code used for this simulation allows the user to choose between second and fourth order accuracy. It is possible to modify it for up to eighth order accuracy using Overture.

To model the Neumann conditions on the boundaries, ghost lines are used. These are additional computational grid points that exist outside of the physical space. They allow one to use the same stencil throughout the computational space, rather than having to write special ones for the side boundaries and corner boundaries.

The first solution to this equation was generated by Onsager using an eigenfunction expansion[2]. This approach generates a semi-analytic, grid independent solution which is free of the physical parameters in the end eigenfunctions. The lateral eigenfunctions can be used to compare similar classes of problems. One of the disadvantages of his approach, as detailed by Wood and Morton, is that it is very difficult to generate higher order eigenfunctions[2]. Another disadvantage is that the solution uses the separation of variables, which means that the boundary conditions must be consistent across entire boundaries. For example, to model feed the center of the centrifuge one would have to model the feed coming across the Stewartson layer over the entire height of the centrifuge. The approach detailed in this section is advantageous because it can model the feed occurring at the appropriate location $z=0.5 Z$ by using an appropriate step function in the boundary conditions.

The boundary conditions for this problem are quite unusual. By formulating the governing equation as a system of three equations, a combination of Neumann and Dirichlet conditions are able to be used. This is advantageous as Overture has built in features for these conditions. Separate features would have to be written to handle the more difficult terms such as $\frac{\partial}{\partial \xi}\left[e^{\xi} \frac{\partial^{2}}{\partial \xi^{2}}\left(e^{\xi} \frac{\partial^{2} \chi}{\partial \xi^{2}}\right)\right]$

The software package Overture is an excellent tool for solving partial differential equations with complex geometries. By using Overture, one is able to increase the order of accuracy very easily. Other desirable features include being able to use a collection of overlapping grids to obtain greater fidelity at areas of interest while being able to use a coarser mesh in less interesting regions. Overture is well suited to handle the implementation while giving the user many options. 


\subsection{Boundary Conditions: Total Reflux Case}

Total reflux is characterized by wheel flow with no mass entering or exiting through the boundaries. This is the simplest case available for analysis. It is useful in that it can be used to test the effects of several different boundary conditions.

Eight boundary conditions are required to solve Equation 37, six in the radial direction and two in the axial direction. For the total reflux case the boundary conditions are given in Table 1. These are essentially the same as those used by Wood and Morton[1,2] with the exception of a slip wall boundary instead of the Ekman condition on the top and bottom. Additional boundary conditions are able to be used, these will be discussed in the future work section.

\begin{tabular}{|l|c|c|}
\hline Physical Interpretation & $\chi$ Statement & Coupled PDE Condition \\
\hline No radial velocity at the rotor wall & $\frac{\partial \chi(0, \eta)}{\partial \xi}=0$ & $\frac{\partial \chi(0, \eta)}{\partial \xi}=0$ \\
\hline No axial velocity at the rotor wall & $\frac{\partial^{2} \chi(0, \eta)}{\partial \xi^{2}}=0$ & $\varphi(0, \eta)=0$ \\
\hline $\begin{array}{l}\text { Fixed axial temperature gradient at } \\
\text { the rotor wall }\end{array}$ & $\frac{\partial}{\partial \xi}\left[e^{\xi} \frac{\partial^{2}}{\partial \xi^{2}}\left(e^{\xi} \frac{\partial^{2} \chi(0, \eta)}{\partial \xi^{2}}\right)\right]=\frac{\rho_{0} \Omega a^{2}}{32(Z / a) A^{10} \epsilon}\left(q^{\prime}+q^{\prime \prime} \eta\right)$ & $\frac{\partial \varrho(0, \eta)}{\partial \xi}=\frac{\rho_{0} \Omega a^{2}}{32(Z / a) A^{10} \epsilon}\left(q^{\prime}+q^{\prime \prime} \eta\right)$ \\
\hline $\begin{array}{l}\text { No radial velocity at the inner } \\
\text { boundary of the Stewartson layer }\end{array}$ & $\frac{\partial \chi\left(\xi_{1}, \eta\right)}{\partial \xi}=0$ & $\frac{\partial \chi\left(\xi_{1}, \eta\right)}{\partial \xi}=0$ \\
\hline $\begin{array}{l}\text { No radial gradient to the axial } \\
\text { velocity at the inner boundary } \\
\text { of the Stewartson layer }\end{array}$ & $\frac{\partial}{\partial \xi}\left(e^{\xi} \frac{\partial^{2} \chi\left(\xi_{1}, \eta\right)}{\partial \xi^{2}}\right)=0$ & $\frac{\partial \varphi\left(\xi \xi_{1}, \eta\right)}{\partial \xi}=0$ \\
\hline $\begin{array}{l}\text { No radial temperature or azimuthal } \\
\text { velocity perturbations at the top } \\
\text { of the atmosphere }\end{array}$ & $\frac{\partial \chi\left(\xi_{1}, \eta\right)}{\partial \eta}=0$ & $\chi\left(\xi_{1}, \eta\right)=0$ \\
\hline $\begin{array}{l}\text { Slip boundary, no axial velocity } \\
\text { at the top of the can }\end{array}$ & $\frac{\partial^{2} \chi\left(\xi, \eta_{1}\right)}{\partial \xi^{2}}=0$ & $\chi\left(\xi, \eta_{1}\right)=\varphi\left(\xi, \eta_{1}\right)=\varrho\left(\xi, \eta_{1}\right)=0$ \\
\hline $\begin{array}{l}\text { Slip boundary, no axial velocity } \\
\text { at the bottom of the can }\end{array}$ & $\frac{\partial^{2} \chi(\xi, 0)}{\partial \xi^{2}}=0$ & $\chi(\xi, 0)=\varphi(\xi, 0)=\varrho(\xi, 0)=0$ \\
\hline
\end{tabular}

Table 1: Boundary Conditions

There is a constant of integration that is obtained from the no radial temperature or azimuthal velocity perturbations at the top of the atmosphere condition. By choosing this value to be zero, that boundary condition is simplified and easier to enforce. Similarly, the conditions on the bottom and top of the centrifuge are able to be reduced to a condition on $\chi$ instead of the second derivative of $\chi$. One should note that the three conditions that are applied to the top and bottom are really different versions of the same condition. They should not result in any inconsistencies.

\subsection{Problem Parameters}

The fluid under consideration for this problem is Uranium Hexafluoride $\mathrm{UF}_{6}$. The other parameters for this simulation are given in Table 2.

\subsection{Choosing A Suitable Grid}

For this problem a grid was chosen that is sufficiently fine to resolve the underlying physics while still running quickly. Originally a rectangular grid was used. However this required a large number of grid points to accurately capture the flow details. Most of the flow effects occur very close to the wall, yet well away from the wall not much is happening. Thus it is desirable to have a very fine scale close to the wall and a coarser one further away from the wall. There are several different options for doing this. One is to use two overlapping grids, which Overture is very well suited to do, with one very fine near the wall and covering only the region near the wall, and a coarse one over the entire solution space. Another option is to employ a stretched grid. This solution is easier to implement and results in a substantial savings in computational 


\begin{tabular}{|l|c|c|}
\hline Parameter & Formula & Value \\
\hline Prandtl number & $\operatorname{Pr}=\frac{\mu C_{p}}{\kappa M}$ & 0.96 \\
\hline Ratio of Specific Heats & $\gamma$ & 1.07 \\
\hline Centrifuge height & $\mathrm{Z}$ & $3.353 \mathrm{~m}$ \\
\hline Centrifuge radius & $\mathrm{a}$ & $9.145 \mathrm{~cm}$ \\
\hline Viscosity & $\mu$ & $10^{-5} \frac{\mathrm{kg}}{\mathrm{ms}}$ \\
\hline Wall speed & $\Omega \mathrm{a}$ & $400,500,700 \mathrm{~m} / \mathrm{s}$ \\
\hline Wall pressure & $P_{w}$ & $13.3 \mathrm{kPa}$ \\
\hline Gas constant & $\mathrm{R}$ & $23.62 \frac{\mathrm{J}}{\mathrm{kgK}}$ \\
\hline Wall density & $\rho_{w}=\frac{P_{w}}{R T_{0}}$ & $1.877 \frac{\mathrm{kg}}{\mathrm{m}^{3}}$ \\
\hline$A^{2}$ & $A^{2}$ & $11.290,17.640,34.575$ \\
\hline Ekman number & $\epsilon=\frac{\mu}{\rho_{w} \Omega a^{2}}$ & $1.456 * 10^{-7}, 1.165 * 10^{-7}, 8.323 * 10^{-8}$ \\
\hline Average Temperature & $T_{0}$ & $300 \mathrm{~K}$ \\
\hline Temperature variation & $\mathrm{dT}$ & $1 \mathrm{~K}$, linear variation \\
\hline
\end{tabular}

Table 2: Simulation parameters for the Rome centrifuge as reported by Wood and Morton [2].

time. Figure 1 shows how a stretched grid concentrates points near the wall. A comparison between an unstretched grid and an exponential stretched grid is given in Figure 2.
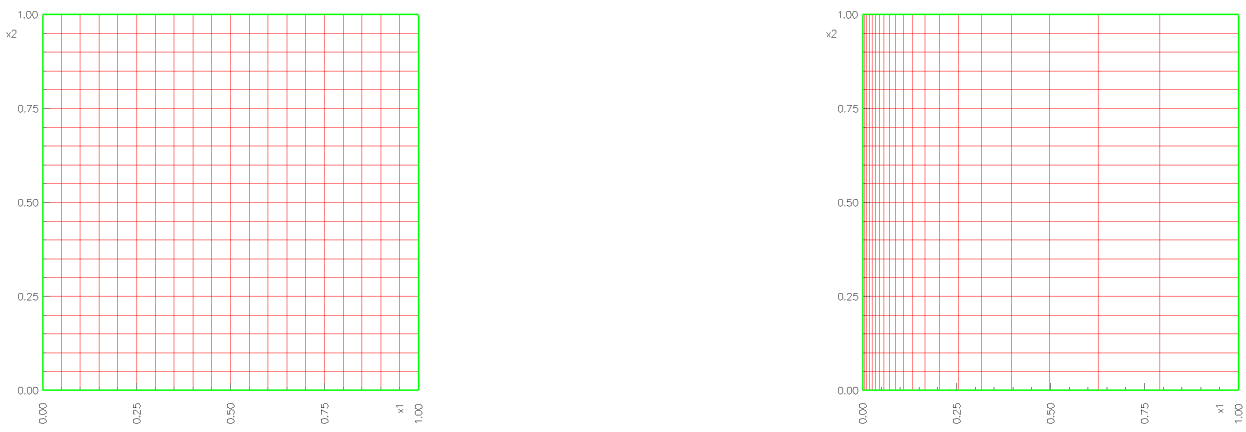

Figure 1: (left) Unstretched Grid. (right) Stretched Grid. The rotor wall is on the left.

The grid that was chosen for this simulation is one with an exponential stretching as shown in Figure 3. The default setting for an exponential stretching in Overture was used. As this was a preliminary analysis, greater fidelity was not required so a stretched grid was deemed suitable. More precise calculations should make use of overlapping grids, especially in when feed is present.

\section{Results}

From each simulation several plots are generated, one for each of the following: master potential, streamlines, axial mass flux, and radial mass flux. The radial and axial mass flux are calculated as follows, respectively:

$$
\begin{aligned}
\rho_{0} v & =\frac{2 A^{2}}{a^{3} Z \sqrt{1-\frac{\xi}{A^{2}}}} \frac{\partial^{2} \chi}{\partial \eta \partial \xi} \\
\rho_{0} u & =\frac{4 A^{4}}{a^{4}} \frac{\partial^{2} \chi}{\partial \xi^{2}}
\end{aligned}
$$

An example test case is shown in Figure 4. These plots are a convenient way to visualize the flow field inside the centrifuge. Figures 5 and 6 are generated to match those obtained by Wood and Morton. The 

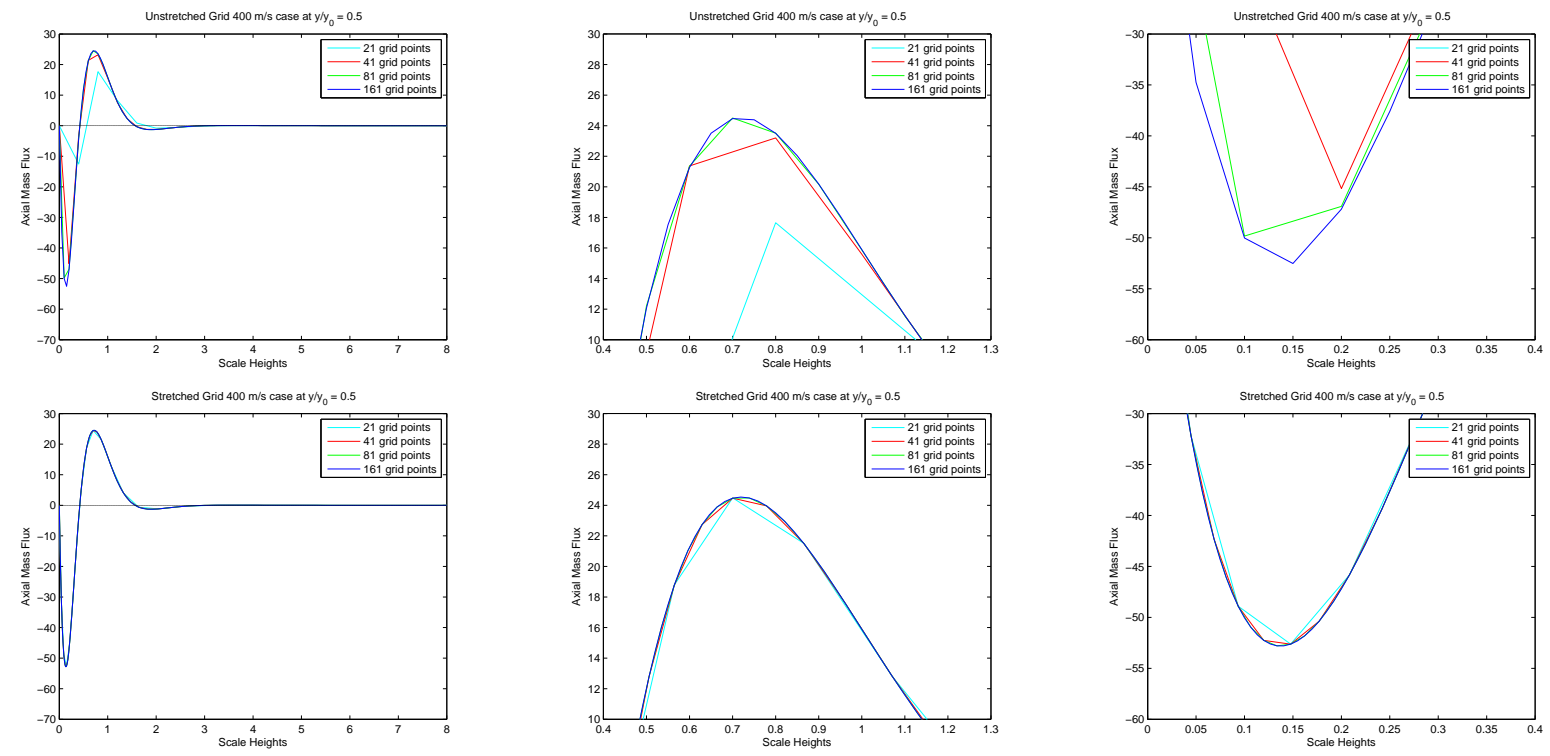

Figure 2: Grid Refinement Study for an Unstretched Grid (top) and a Stretched Grid (bottom). (left) Entire Domain. (middle) Close-up of Top Peak. (right) Close-up of Bottom Peak.

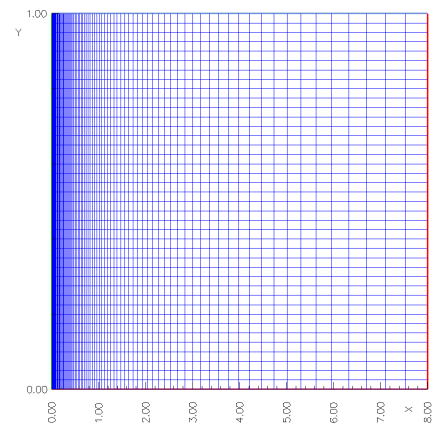

Figure 3: Computational Grid. 
figures show a reasonable match, within ten percent, and follow the same qualitative trends. They are not expected to match precisely because the conditions used by Wood and Morton are not known exactly. Also, the results shown by Wood and Morton contain the Ekman condition which was not used in this preliminary analysis. Overall the agreement between the two simulations is substantial.
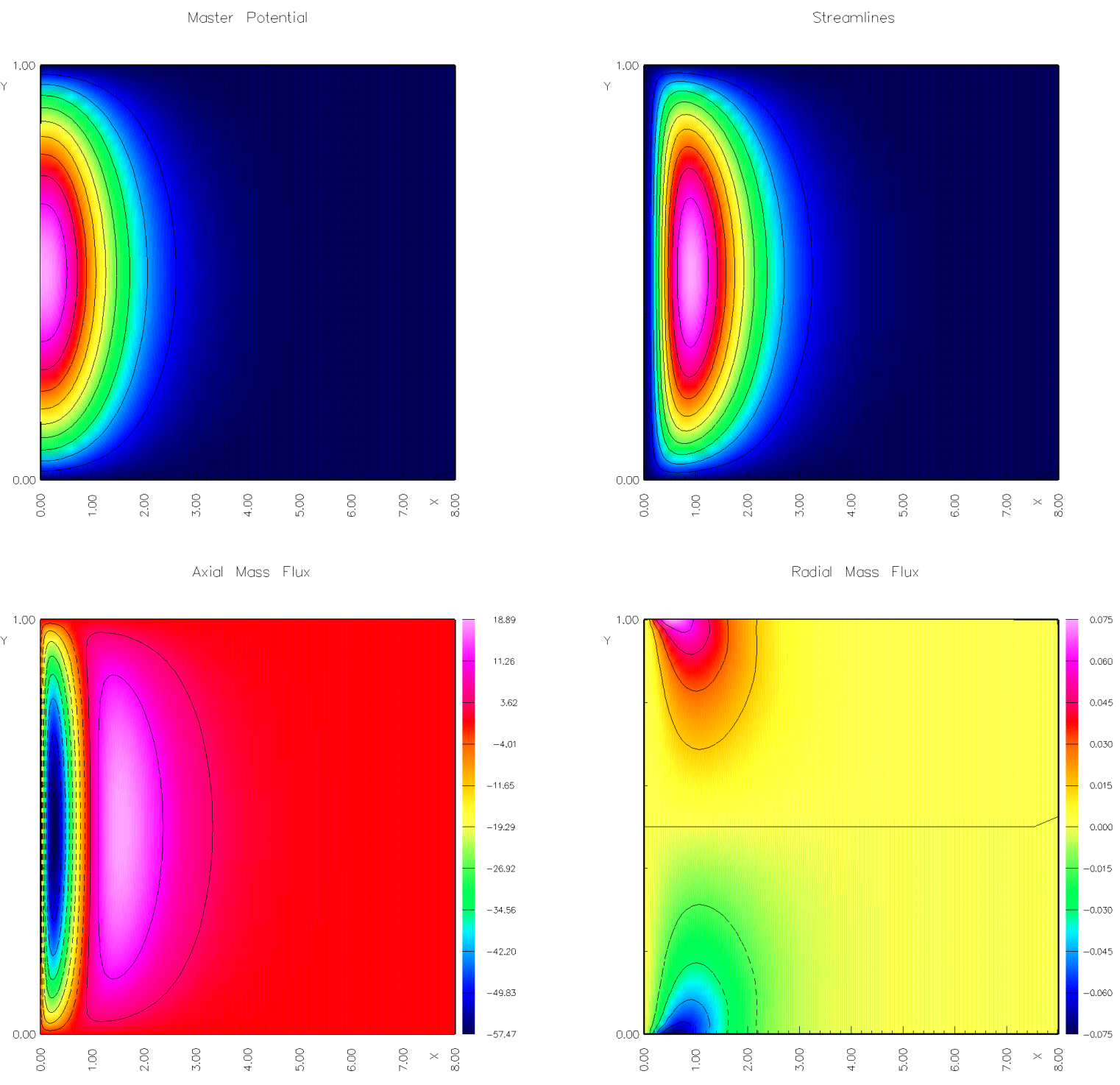

Figure 4: Simulation Graphical Outputs for a Centrifuge with a Wall Speed of $500 \mathrm{~m} / \mathrm{s}$. (top left) Master Potential. (top right) Streamlines. (bottom left) Axial Mass Flux $\left[\rho_{0} u, \frac{g}{m^{2} s}\right]$. (bottom right) Radial Mass Flux $\left[\rho_{0} v, \frac{g}{m^{2} s}\right]$. Note that the horizontal scale is scale heights, $\xi$ and the rotor wall is on the left.

\subsection{Verification}

Manufactured solutions are one of the tools being used to verify this code. The basic idea behind a manufactured solution is to pick a known analytic function to be the solution to the problem. The boundary conditions and the equation are forced to ensure that the code produces the desired solution. The solution obtained from the code is compared to the analytic function to determine the error. This can be used to verify that the code is working properly. Errors that are not obvious from looking at a solution can be 

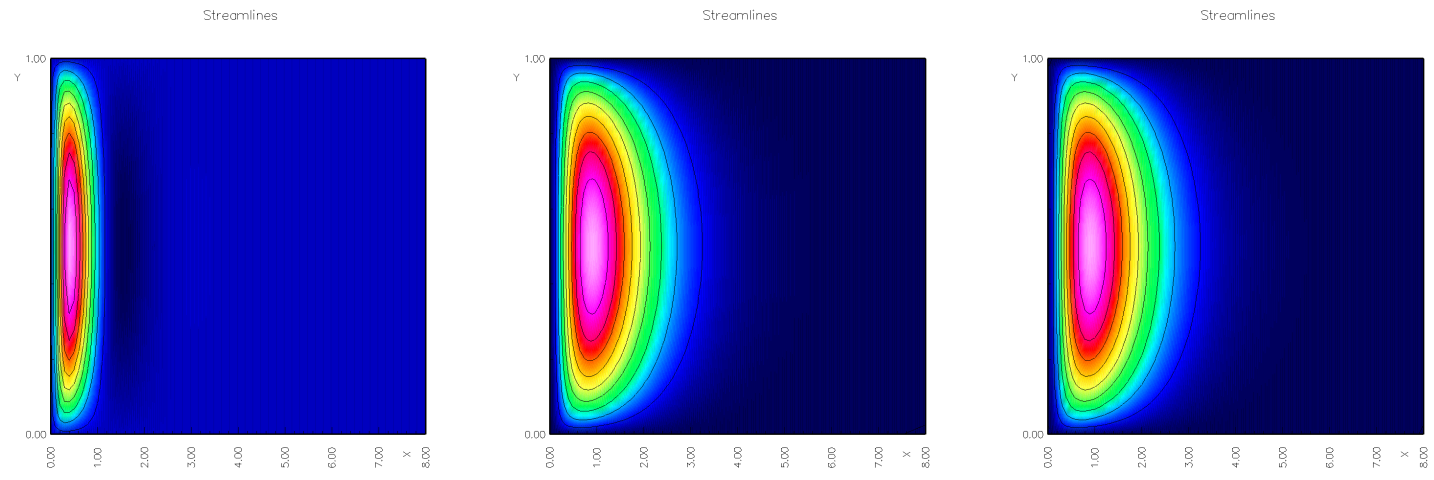

Figure 5: Streamlines for a Centrifuge with varying Wall Speed. (left) $400 \mathrm{~m} / \mathrm{s}$. (middle) $500 \mathrm{~m} / \mathrm{s}$. (right) $700 \mathrm{~m} / \mathrm{s}$. Note that the horizontal scale is scale heights, $\xi$, which are a relative term that varies with the wall speed. If one were to do the graphs with absolute radial position on the horizontal scale they would appear essentially the same, all with the fluid motion occurring at the rotor wall. Note that the rotor wall is on the left.
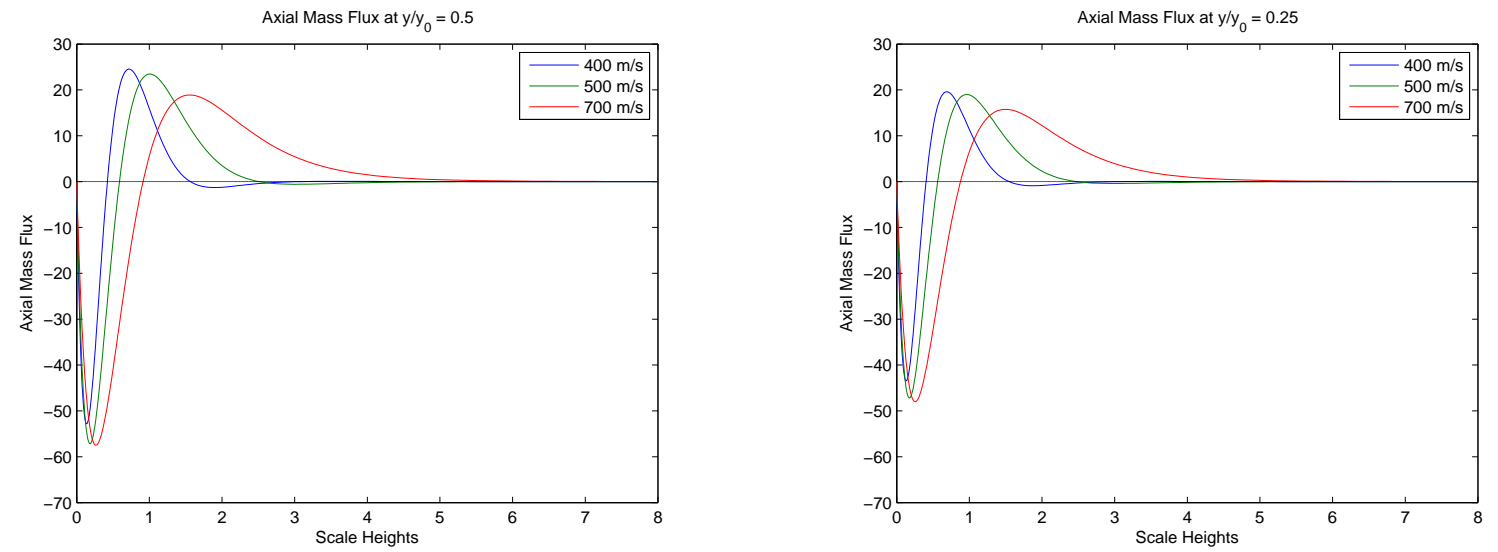

Figure 6: (left) Axial Mass Flux at 0.5 $\eta_{1}$. (right) Axial Mass Flux at $0.25 \eta_{1}$. The rotor wall is on the left. 
revealed by techniques such as checking actual error convergence rates to see if they match the intended rates.
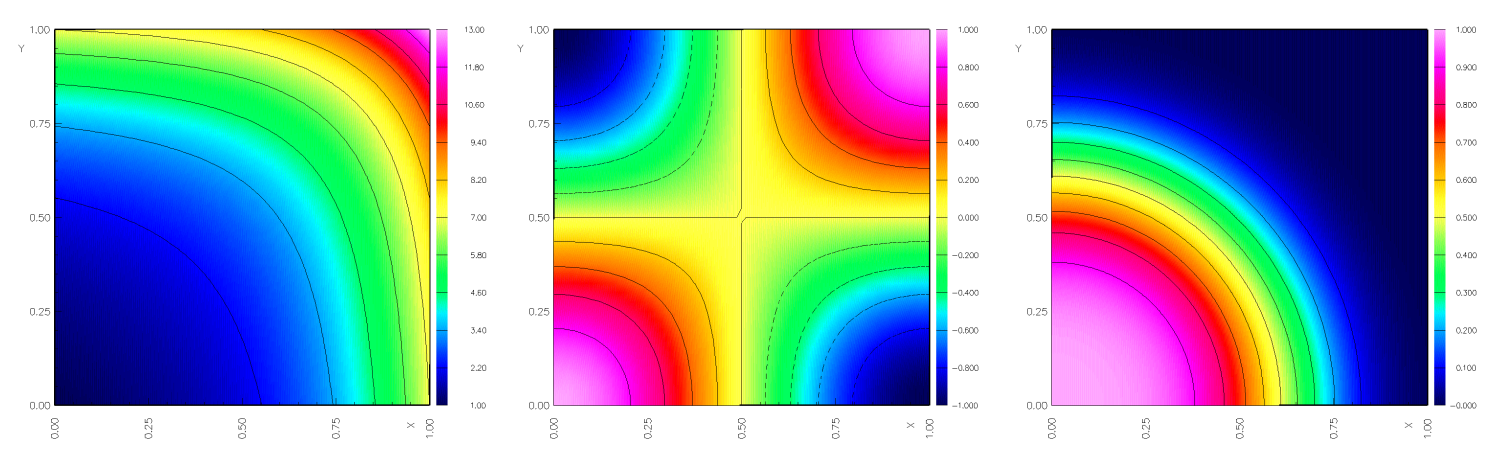

Figure 7: Exact Solutions Used for Convergence Study. (left) Polynomial Function. (middle) Trigonometric Function. (right) Pulse Function.

For each of the given convergence tables in Table 5, the convergence rate was approximated using a least squares fit of the log average grid spacing vs. the log of the maximum absolute error. They are each from the the $500 \mathrm{~m} / \mathrm{s}$ wall speed case evaluated on the actual computational grid shown in Figure 3 .

\subsection{Validation}

Reliable experimental data is difficult to obtain and is not expected to be available for validation. A reasonable amount of information is known about the flow inside a gas centrifuge to use physical insight to validate the code. One of the features that is expected is a weak countercurrent flow. This flow pattern is easily seen in the streamline of Figure 5. Also by looking at the mass flux, which is the velocity multiplied by the density, one can verify that the radial component of the flow is strongest at the top and bottom of the machine. The flow has an equal and opposite velocity at the top and bottom of the machine. A similar phenomenon can be observed in the case of axial mass flux, however in that case the magnitudes differ due to the density variation. One could integrate over the flow area to verify that the net flow rate is zero. Figure 4 illustrates both of the above mentioned flow patterns.

\subsection{Known Errors}

There are still several areas of work that are needed to complete this project. While a fourth order accurate scheme is being used, the rate of convergence given by the manufactured solution does not match the expected value. The maximum error values obtained are very large which is a cause for concern. As the three equations are all coupled, error propagates through each one of the components and can become very large as shown in Figure 8. It appears that the largest error is located at the corners as is often the case.

The simulation behaved as expected in the 1-D radial case yet has had difficulty incorporating the axial derivative terms. This error is reduced as the wall speed increases since a higher wall speed results in a lower value of the constant term in front of the second axial derivative.

Another difficulty is that occasionally the external grids that are loaded into the simulation are not recognized by the Overture database. This is easily fixed by re-creating one of the grids.

\section{Conclusion}

While there still remains work to be done a reasonable baseline solution has been obtained for the total reflux case. The results obtained in this simulation are in reasonable agreement with the results obtained by Wood and Morton. Further work to eliminate the corner errors and to achieve the desired order of accuracy is required. Once this is done greater complexity can be introduced. 


\begin{tabular}{|c|c|c|c|}
\hline $\mathrm{h}$ & $\left|\chi-\chi_{e}\right|_{\infty}$ & $\left|\varphi-\varphi_{e}\right|_{\infty}$ & $\left|\varrho-\varrho_{e}\right|_{\infty}$ \\
\hline 0.40 & $2.89 \mathrm{e}+03$ & $9.41 \mathrm{e}+05$ & $3.37 \mathrm{e}+08$ \\
\hline 0.20 & $1.40 \mathrm{e}+02$ & $3.63 \mathrm{e}+04$ & $3.12 \mathrm{e}+07$ \\
\hline 0.10 & $1.08 \mathrm{e}+01$ & $3.32 \mathrm{e}+03$ & $2.89 \mathrm{e}+06$ \\
\hline 0.05 & $7.50 \mathrm{e}+00$ & $2.30 \mathrm{e}+02$ & $2.00 \mathrm{e}+05$ \\
\hline \hline rate & 3.95 & 3.94 & 3.56 \\
\hline
\end{tabular}

Table 3: Convergence Rate Test: Polynomial Function.

\begin{tabular}{|c|c|c|c|}
\hline $\mathrm{h}$ & $\left|\chi-\chi_{e}\right|_{\infty}$ & $\left|\varphi-\varphi_{e}\right|_{\infty}$ & $\left|\varrho-\varrho_{e}\right|_{\infty}$ \\
\hline 0.40 & $7.61 \mathrm{e}+01$ & $4.08 \mathrm{e}+04$ & $1.55 \mathrm{e}+07$ \\
\hline 0.20 & $1.50 \mathrm{e}-00$ & $5.34 \mathrm{e}+02$ & $1.80 \mathrm{e}+05$ \\
\hline 0.10 & $1.27 \mathrm{e}-01$ & $6.24 \mathrm{e}-00$ & $2.63 \mathrm{e}+04$ \\
\hline 0.05 & $5.63 \mathrm{e}-02$ & $2.71 \mathrm{e}+01$ & $2.36 \mathrm{e}+04$ \\
\hline \hline rate & 3.48 & 3.81 & 3.09 \\
\hline
\end{tabular}

Table 4: Convergence Rate Test: Trigonometric Function.

\begin{tabular}{|c|c|c|c|}
\hline $\mathrm{h}$ & $\left|\chi-\chi_{e}\right|_{\infty}$ & $\left|\varphi-\varphi_{e}\right|_{\infty}$ & $\left|\varrho-\varrho_{e}\right|_{\infty}$ \\
\hline 0.40 & $5.00 \mathrm{e}-03$ & $7.15 \mathrm{e}-02$ & $1.07 \mathrm{e}+01$ \\
\hline 0.20 & $3.17 \mathrm{e}-04$ & $3.83 \mathrm{e}-03$ & $5.26 \mathrm{e}-01$ \\
\hline 0.10 & $2.24 \mathrm{e}-05$ & $2.53 \mathrm{e}-04$ & $3.75 \mathrm{e}-02$ \\
\hline 0.05 & $2.58 \mathrm{e}-06$ & $1.73 \mathrm{e}-05$ & $2.41 \mathrm{e}-03$ \\
\hline \hline rate & 3.66 & 4.00 & 4.02 \\
\hline
\end{tabular}

Table 5: Convergence Rate Test: Pulse Function. 

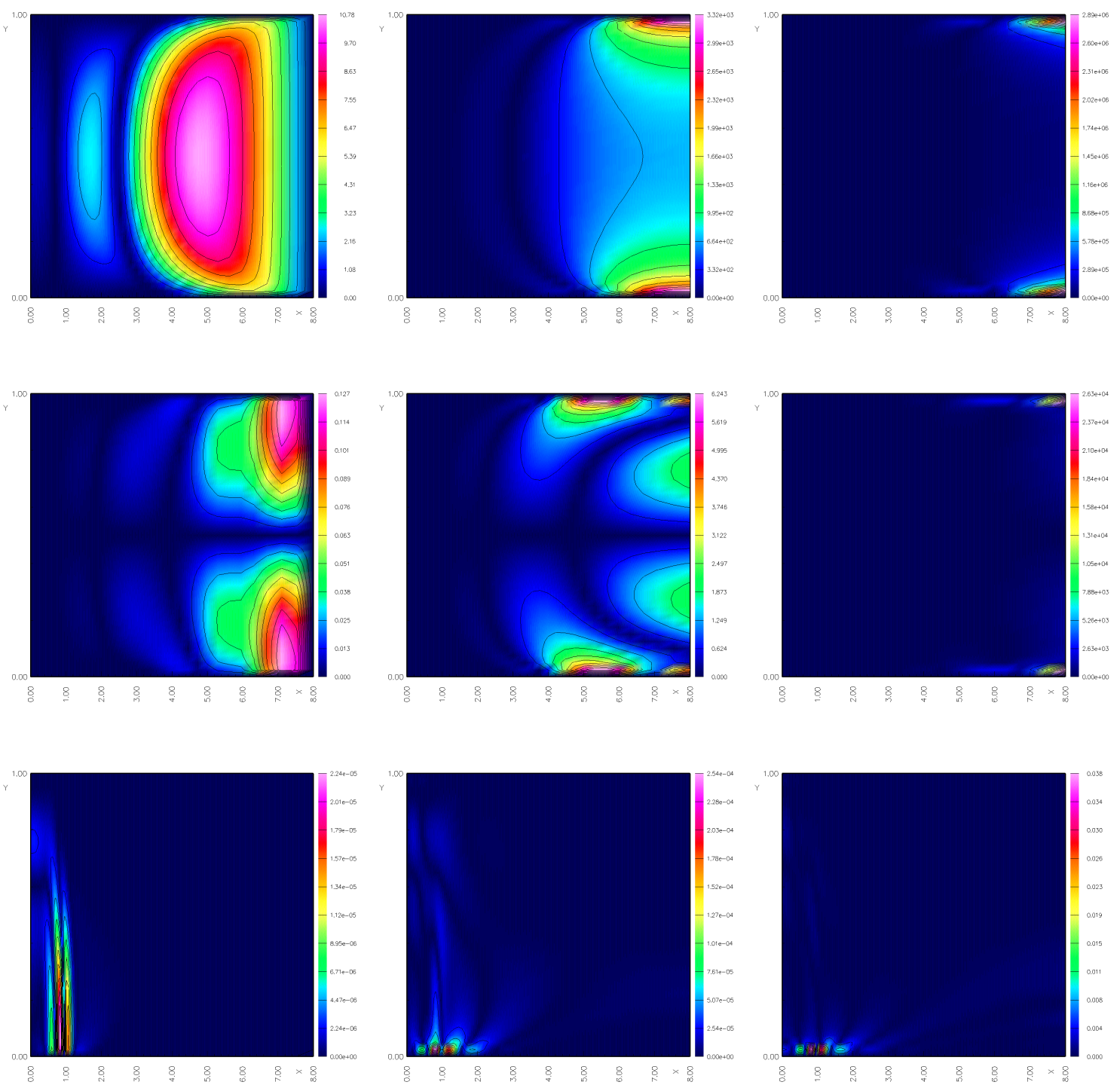

Figure 8: Error From Manufactured Solution Test. (top) Polynomial Function. (middle) Trigonometric Function. (bottom) Pulse Function. (left column) $\chi$ error. (middle column) $\varphi$ error. (right column) $\varrho$ error. Note that the horizontal scale is scale heights, $\xi$. 


\section{Future Work}

Once the current difficulties with incorporating the axial derivative term are solved there are a variety of areas in which further progress can be made. A logical first step would be to replace the slip condition at the top and bottom with the Ekman condition. This will provide insight into just how much of an effect the Ekman condition has on the final solution. The results from Wood and Morton are also available for verification. Once the Ekman condition is implemented, the code should be modified to accept overlapping grids. This will allow the user to concentrate points at areas of interest, which is especially of interest when there are feed, product, and waste streams. An overlapping grid has the advantage of using a coarse grid for speed over the entire domain and finer grids over only the areas of interest. It would also be instructive to run the simulation with several different sizes for the solution space to see what impact the size of the computational space has on the solution. A similar task would be to perform a sensitivity study for the different variables in Onsager's Master Equation. The solution is currently obtained with scale heights as the radial scale, it is desirable to also get the solution in physical space, especially for comparison with other simulations.

Once the code is working with overlapping grids, it is desirable to move from the highly idealized total reflux case to a more realistic case with feed, product, and waste streams. This scenario is much closer to what actually occurs inside a gas centrifuge and introduces its own complexities, such as how to handle the different properties of the product and waste streams. One could also add scoop drive and end cap thermal drive to the wall thermal drive. This would most easily be accomplished by including source and sink terms as done by Wood and Morton[2]. Another useful tool would be to have an eigenfunction solution for comparison with the numerical one. This provides an additional check to make sure that the code is working correctly.

Separative Work (SWU) is the quantity that is most desired for the analysis of a gas centrifuge. The SWU and feed rate are the two most important variables for doing calculations for centrifuge cascades, which are always used in practice. To obtain the SWU, the species equation must also be solved. It would also be useful to modify the code to solve for the temperature field. The Cohen M-factor provides a measure of how different a centrifuge is operating from ideal at each point in the axial direction.

One could also make a graphical user interface (GUI) that would make it easier for a user to customize a simulation. For example, one could quickly run a simulation of the total reflux case and then compare that solution to the one obtained in the more realistic with feed case. This tool would make the code much easier for a non-expert, as well as reduce the amount of time it takes to learn to use the program.

The results obtained from this program could be used as an initial guess for a more complex Navier-Stokes code. This would provide such a code with a reasonable estimate of the flow field in the axisymmetric plane. It remains to be determined whether such a high-fidelity Navier-Stokes simulation is needed.

\section{References}

[1] Donald R. Olander. The theory of uranium enrichment by the gas centrifuge. Progress in Nuclear Energy, 8:1-31, 1981.

[2] Houston G. Wood and J.B. Morton. Onsager's pancake approximation for the fluid dynamics of a gas centrifuge. Journal of Fluid Mechanics, 101(1):1-31, 1980.

\section{A Pseudocode for Simulation}

This simulation was run from the file "Onsagerspancakeapproxtotalrefluxcase.C" It was written in C++ using the Overture library.

Pseudocode for "Onsagerspancakeapproxtotalrefluxcase.C"

1. Include relevant libraries

2. Choose whether to plot output (set doplot to 1 for yes, 0 for no)

3. Choose whether to recordsteps in the plot window (recordsteps to 0 for no, 1 for yes)

4. Ask user for inputs (wall speed, grid file name, number of horizontal grid points, number of vertical grid 
points, forcing option).

5. Load grid

6. Define Simulation parameters

7. Update mapped grid properties (unnecessary if grid is loaded, required if a grid is generated within file)

8. Create forcing functions

9. Initialize coefficient matrix: coeff

10. Initialize grid functions

11. Name grid functions

12. Define mapped grid operators

13. Define coefficient matrix over entire computational space

14. Define ghostlines for coefficient matrix

15. Define boundary conditions for coefficient matrix

16. Initialize right hand side over the entire computational space

17. Define right hand side on the boundaries

18. Initialize solver

19. Simultaneously solve the system of equations

20. Extract streamlines

21. Extract radial and axial mass flow rate

22. Calculate error all over for twilight zone flows

23. Find maximum error for each of the three equations (from system)

24. Open plot window (if chosen above)

25. Record steps in plot GUI (if chosen above)

26. Plot master potential, error, streamlines, axial and radial mass flux (if chosen above)

27. End 